\title{
The Numerical Simulation of Properties with High Angular Speed \& Low Angular Acceleration in Three \& Five Freedoms of Robotic Arm I
}

Run $\mathrm{Xu}^{1 *}$

${ }^{T}$ Gyeongsang National University, Metallurgical Engineering Dept., Chinju 52828, Korea

*Corresponding Author: Run Xu

Gyeongsang National University, Metallurgical Engineering Dept., Chinju 52828, Korea

\section{Article History}

Received: 03.12.2021

Accepted: 08.01.2022

Published: 12.01.2022

\begin{abstract}
In robot design and application the force and angle with angular speed is important so this study will model numerical simulation and discuss detail data to investigate their property. The force may increase as arm 1 angle increases whilst it may increase if angular speed increases in three freedoms. Meantime it will decrease if angular acceleration increases. It is found that with the angular speed increasing all three forces may increase whilst the angular acceleration will cause its increase too in five freedoms. From this value it is observed that $\mathrm{F}_{2}$ is prior one to ensure the strength and fatigue life then $\mathrm{F}_{2}$ is second one to estimate its strength whilst $\mathrm{F}_{3}$ may be neglected. The force may increase as arm 1 angle increases whilst it may increase if angular speed increases in three freedoms. Meantime it will decrease if angular acceleration increases. There is big distance to attain $5 \mathrm{KN}$ between the conditions. The effective factor turn to the force is $F_{1}>F_{3}>F_{2}$ in three freedoms. The force may increase from $15 \mathrm{~N}$ to $15 \mathrm{KN}$ and $18 \mathrm{KN}$ with $\mathrm{F}_{3}, \mathrm{~F}_{2}$ and $\mathrm{F}_{1}$ in five freedoms. Among them $F_{3}$ is the least one and $F_{1}$ is the biggest one. The effect factor turn is $F_{1}>F_{2}>F_{3}$. so the $F_{1}$ and $F_{2}$ is important one while $\mathrm{F}_{3}$ is neglected.
\end{abstract}

Keywords: Numerical simulation; force and angle; angular speed \& acceleration; robotic arm; angular Acceleration; three \& five freedoms.

\section{INTRODUCTION}

In recent the robotic arm has been applied to many occasion in factory which can help people to work in difficult, dirty and dangerous place so its application will be more prevailed in future which is estimated in this study. China has been the largest country which can own the largest domestic market in the world, but it has the third market occupant since it has not owned the enough advanced condition [1-5]. So that we must positively construct the clean and criteria house to meet the demand for robotic arm to ensure precise work.

In robotic design the force is an important factor to consider since the strength must meet demand no matter what it may work in factory $[6,7]$. So that according to the function it may be designed to satisfy the no fracture and longer fatigue life for its long life and high load to work in automatic flow line to be substituted to human worker. The biggest one will destination in this paper and how to save manufacture cost is the second one. So it is needed that the mass and load may become first thing to prepare; secondly for the cost decrease the redundant load shall be prohibited.

In this paper the condition is changed like angular speed and acceleration to observe the three forces status to ensure security of strength and save cost status. The three freedoms and five ones are investigated in this paper detail with parameters like force and angle with angular speed and acceleration. We try to find the various condition of effective factor in order to search intrinsic properties relationship which is the destination in this paper.

In short the properties are searched through parameters in detail. We look forward to finding new change with force and speed $\&$ acceleration for further research.

Copyright (C) 2022 The Author(s): This is an open-access article distributed under the terms of the Creative Commons Attribution 4.0 International License (CC BY-NC 4.0) which permits unrestricted use, distribution, and reproduction in any medium for non-commercial use provided the original author and source are credited. 


\section{Numerical Simulations}

In Figure 1 there are three freedoms in mechanical arm that name as $1 \sim 3$. Meantime there are two other ones call $4 \& 5$ which is included in five freedoms as a rotational and crawling function. In Figure 1 the schematic shows the simplified principle of robot. The coordinate XAY is three freedoms and $\mathrm{X}^{\prime} \mathrm{A}^{\prime} \mathrm{Y}$ it five freedoms. In this study the five freedoms not three one is deduced since it is complicated.

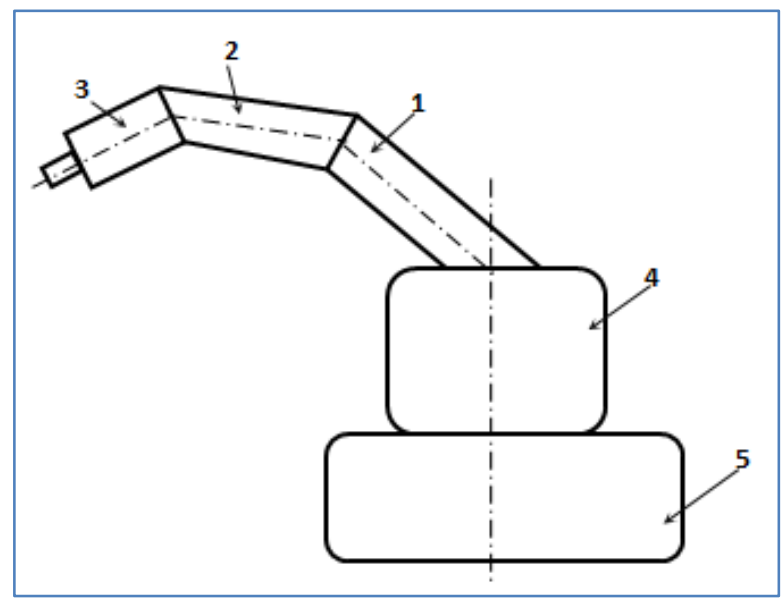

Fig-1: Construction schematic of mechanical arm in series in robot 3-hand part; 2-wrist part;1-arm part; 4-waist part; 5-two crawling wheel

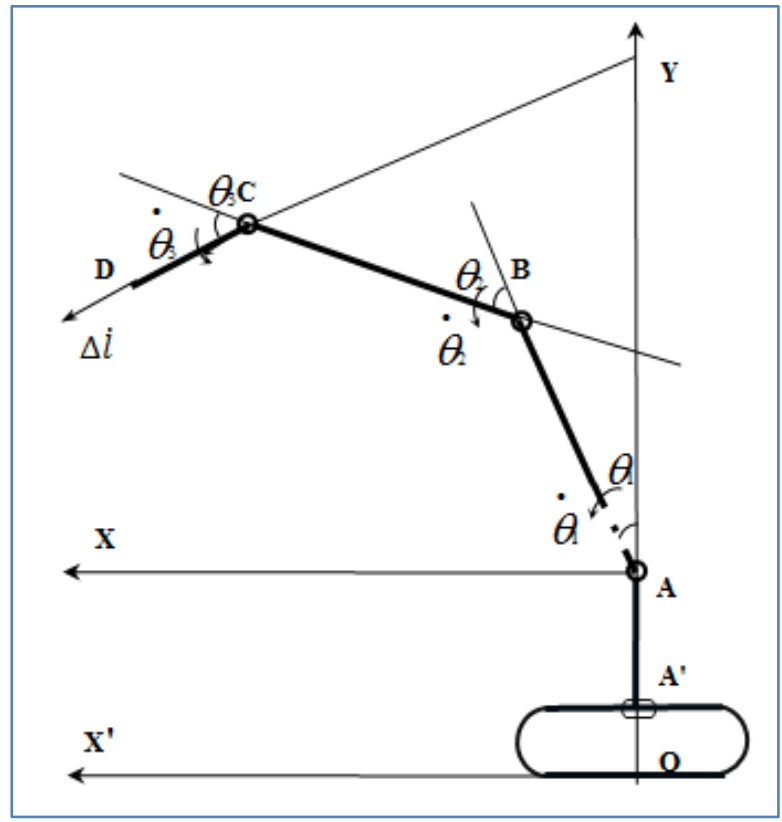

Fig-2: Principle schematic of mechanical arm in series in robot

The system kinetic energy is $[1,3]$

$$
E_{k}=\frac{1}{2} \sum_{i}^{n}\left(m_{1} v_{1}^{2}+m_{2} v_{2}^{2}+m_{3} v_{3}^{2}\right)
$$

Here $m_{\mathrm{i}}$ : mass of i component ; $J_{s i}$ : rotary inertia of i component relative to center of mass; $v_{s}:$ center of mass in $\mathrm{i}$ component; $\omega_{i}$ : angular velocity in i component; $v_{1}, v_{2}$ and $v_{3}$ is 1,2 and 3 velocities respectively. 
$v_{D}=\sqrt{\dot{X}_{D}^{2}+\dot{Y}_{D}^{2}}$

From Figure 2 it is known that position coordinate below $\left\{\begin{array}{l}X_{D}=\vec{l}_{1} \sin \vartheta_{1}+\vec{l}_{2} \sin \left(\theta_{1}+\theta_{2}\right)+\vec{l}_{3} \sin \left(\theta_{1}+\theta_{3}+\theta_{3}\right) \\ Y_{D}=\left(\vec{l}_{1}+\vec{l}_{4}\right) \cos \theta_{1}+\left(\vec{l}_{2}+\vec{l}_{4}\right) \cos \left(\theta_{1}+\theta_{2}\right)+\left(\vec{l}_{3}+\vec{l}_{4}\right) \cos \left(\theta_{1}+\theta_{2}+\theta_{3}\right)\end{array}\right.$

Derivating the equations we gain the $\dot{X}_{c}, \dot{Y}_{c}$ and $\dot{X}_{3}$ velocity in hand , $\dot{\theta}_{1}, \dot{\theta}_{2}$ and $\dot{\theta}_{3}$ one in joints. Suppose that the acceleration is $\ddot{\theta}_{1}, \ddot{\theta}_{2}$ and $\ddot{\theta}_{3}$ and the angular acceleration is $\ddot{\omega}_{1}, \ddot{\omega}_{2}$ and $\ddot{\omega}_{3}$ in joints.

$\left\{\begin{array}{l}\dot{X}_{\mathrm{D}}=\dot{\theta}_{1} \vec{l}_{1} \cos \theta_{1}+\left(\dot{\theta}_{1}+\dot{\theta}_{2}\right) \vec{l}_{2} \cos \left(\theta_{1}+\theta_{2}\right)+\left(\dot{\theta}_{1}+\dot{\theta}_{2}+\dot{\theta}_{3}\right) \vec{l}_{3} \cos \left(\theta_{1}+\theta_{2}+\theta_{3}\right) \\ \dot{Y}_{\mathrm{D}}=\dot{\theta}_{1}\left(\vec{l}_{1}+\vec{l}_{4}\right) \sin \theta_{1}+\left(\dot{\theta}_{1}+\dot{\theta}_{2}\right)\left(\vec{l}_{2}+\vec{l}_{4}\right) \sin \left(\theta_{1}+\theta_{2}\right)+\left(\dot{\theta}_{1}+\dot{\theta}_{2}+\dot{\theta}_{3}\right)\left(\vec{l}_{3}+\vec{l}_{4}\right) \sin \left(\theta_{1}+\theta_{2}+\theta_{3}\right)\end{array}\right.$

$v_{B}, v_{C}$ and $v_{D}$ is $\mathrm{B}, \mathrm{C}$ and $\mathrm{D}$ velocities respectively. So $\mathrm{D}$ point velocity is

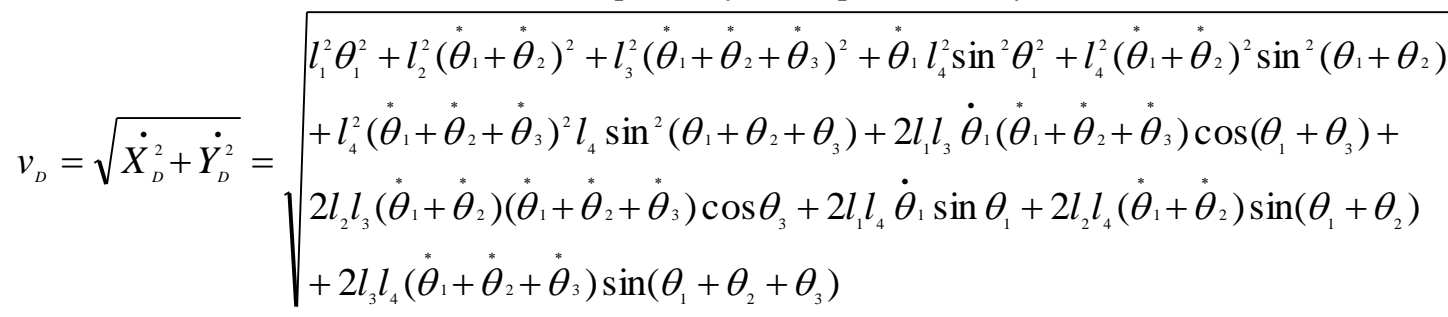

C point velocity is

$v_{c}=\sqrt{\dot{X}_{c}^{2}+\dot{Y}_{c}^{2}}=\sqrt{l_{1}^{2} \theta_{1}^{2}+l_{2}^{2}\left(\ddot{\theta}_{1}+\ddot{\theta}_{2}\right)^{2}-2 l_{1} l_{2} \dot{\theta}_{1}\left(\ddot{\theta}_{1}+\ddot{\theta}_{2}\right) \cos \theta_{2}}$

$v_{B}=\vec{l}_{1} \dot{\theta}_{1}$

Substituting two equations above to equation below

$E_{K}=\frac{1}{2} \vec{l}_{1}\left(\vec{l}_{1}+\vec{l}_{4}+\vec{l}_{5}\right)\left(m_{1}+m_{2}+m_{3}\right) \theta_{1}^{2}+\frac{1}{2} \vec{l}_{2}\left(\vec{l}_{2}+\vec{l}_{4}+\vec{l}_{5}\right) m_{2}\left(\theta_{1}+\theta_{2}\right)^{2}+\frac{1}{2} \vec{l}_{2}\left(\vec{l}_{2}+\vec{l}_{4}+\vec{l}_{5}\right) m_{2}\left(\dot{\theta}_{1}+\dot{\theta}_{2}\right)^{2}$ $+\frac{1}{2} \vec{l}_{3}\left(\vec{l}_{3}+\vec{l}_{4}+\vec{l}_{5}\right) m_{3}\left(\theta_{1}+\theta_{2}+\theta_{3}\right)^{2}+2 \vec{l}_{4}^{2} m_{3} \dot{\theta}_{1} \sin ^{2} \theta_{2}+\vec{l}_{4}^{2} m_{3} \dot{\theta}_{1}\left(\dot{\theta}_{1}+\dot{\theta}_{2}\right)^{2} \sin ^{2}\left(\theta_{1}+\theta_{2}\right)+\vec{l}_{4}^{2} m_{3}\left(\dot{\theta}_{1}+\dot{\theta}_{2}\right.$

$\left.+\dot{\theta}_{3}\right)^{2} \sin ^{2}\left(\theta_{1}+\theta_{2}+\theta_{3}\right)+2 \vec{l}_{1} \vec{l}_{2} m_{2} \dot{\theta}_{1}\left(\dot{\theta}_{1}+\dot{\theta}_{2}\right) \cos \theta_{2}+\vec{l}_{1} \vec{l}_{2} m_{3} \dot{\theta}_{1}\left(\dot{\theta}_{1}+\dot{\theta}_{2}+\dot{\theta}_{3}\right) \cos \left(\theta_{1}+\theta_{2}\right)+\vec{l}_{2} \vec{l}_{3} m_{3}\left(\dot{\theta}_{1}\right.$

$\left.+\dot{\theta}_{2}\right)\left(\dot{\theta}_{1}+\dot{\theta}_{2}+\dot{\theta}_{3}\right) \cos \theta_{3}+\frac{1}{2}\left(\vec{l}_{4}+\vec{l}_{5}\right)\left(m_{1}+m_{2}+m_{3}\right) \theta_{1}^{2}+\frac{1}{2}\left(\vec{l}_{4}+\vec{l}_{5}\right) m_{2}\left(\theta_{1}+\theta_{2}\right)^{2}+\frac{1}{2}\left(\vec{l}_{4}+\vec{l}_{5}\right) m_{2}\left(\dot{\theta}_{1}+\right.$

$\left.\dot{\theta}_{2}\right)^{2}+2 \vec{l}_{1} \vec{l}_{4} m_{3} \dot{\theta}_{1} \sin \left(\theta_{1}+\theta_{2}\right)+2 \vec{l}_{1} \vec{l}_{4} m_{3}\left(\dot{\theta}_{1}+\dot{\theta}_{2}\right) \sin \left(\theta_{1}+\theta_{2}\right)+2 \vec{l}_{3} \vec{l}_{4} m_{3}\left(\dot{\theta}_{1}+\dot{\theta}_{2}+\dot{\theta}_{3}\right) \sin \left(\theta_{1}+\theta_{2}+\theta_{3}\right)$

Here

$\frac{\partial E_{K}}{\partial \dot{\theta}_{1}}=\left(\dot{\theta}_{1}+\dot{\theta}_{2}\right) \vec{l}_{2} m_{2}+2 \vec{l}_{4}^{2} m_{3} \sin ^{2} \theta_{2}+2\left(\dot{\theta}_{1}+\dot{\theta}_{2}\right)^{2} \vec{l}_{4}^{2} m_{3} \sin ^{2}\left(\theta_{1}+\theta_{2}\right)+2$

$\dot{\theta}_{1}\left(\dot{\theta}_{1}+\dot{\theta}_{2}\right) \vec{l}_{4}^{2} m_{3} \sin ^{2}\left(\theta_{1}+\theta_{2}\right)+2 \vec{l}_{4} m_{3} \sin ^{2}\left(\theta_{1}+\theta_{2}+\theta_{3}\right)+2\left(\dot{\theta}_{1}+\dot{\theta}_{2}\right) \vec{l}_{1} \vec{l}_{2}$

$m_{2} \cos \theta_{2}+2 \dot{\theta}_{1} \vec{l}_{1} \vec{l}_{2} m_{2} \cos \theta_{2}+\dot{\theta}_{1} \vec{l}_{1} \vec{l}_{2} m_{3} \cos \left(\theta_{1}+\theta_{2}\right)+\vec{l}_{1} \vec{l}_{2} m_{3}\left(\dot{\theta}_{1}+\dot{\theta}_{2}+\right.$

$\left.\dot{\theta}_{3}\right) \cos \left(\theta_{1}+\theta_{2}+\theta_{3}\right)+\vec{l}_{2} m_{3}\left(\dot{\theta}_{1}+\dot{\theta}_{2}\right) \cos \theta_{3}+\vec{l}_{2} \vec{l}_{3} m_{3}\left(\dot{\theta}_{1}+\dot{\theta}_{2}+\dot{\theta}_{3}\right) \cos \theta_{3}$

$+2 \vec{l}_{1} \vec{l}_{4} m_{3} \sin \left(\theta_{1}+\theta_{2}\right)+2 \vec{l}_{1} \vec{l}_{4} m_{3} \sin \left(\theta_{1}+\theta_{2}\right)+2 \vec{l}_{1} \vec{l}_{4} m_{3} \cos \left(\theta_{1}+\theta_{2}+\theta_{3}\right)$ 
$\frac{\partial E_{K}}{\partial \dot{\theta}_{2}}=\vec{l}_{2} m_{2}\left(\dot{\theta}_{1}+\dot{\theta}_{2}\right)+\vec{l}_{4} m_{3}\left(\dot{\theta}_{1}+\dot{\theta}_{2}\right)^{2} \sin ^{2}\left(\theta_{1}+\theta_{2}\right)+2 \vec{l}_{4} m_{3} \dot{\theta}_{1}\left(\dot{\theta}_{1}+\dot{\theta}_{2}\right) \sin$

${ }^{2}\left(\theta_{1}+\theta_{2}\right)+2 \vec{l}_{4} m_{3}\left(\dot{\theta}_{1}+\dot{\theta}_{2}+\dot{\theta}_{3}\right) \sin ^{2}\left(\theta_{1}+\theta_{2}\right)+2 \vec{l}_{1} \vec{l}_{2} m_{2} \dot{\theta}_{1} \cos \theta_{2}+\vec{l}_{1} \vec{l}_{2} m_{2}$

$\left(\dot{\theta}_{1}+\dot{\theta}_{2}\right) \cos \theta_{2}+\vec{l}_{2} \vec{l}_{3} m_{3}\left(\dot{\theta}_{1}+\dot{\theta}_{2}+\dot{\theta}_{2}\right)+\cos \left(\theta_{1}+\theta_{2}\right)+\vec{l}_{2} \vec{l}_{3} m_{3}\left(\dot{\theta}_{1}+\dot{\theta}_{2}+\dot{\theta}_{3}\right)$

$\cos \theta_{3}+\vec{l}_{2} \vec{l}_{3} m_{3}\left(\dot{\theta}_{1}+\dot{\theta}_{2}\right) \cos \theta_{3}+2 \vec{l}_{1} \vec{l}_{4} m_{3} \sin \left(\theta_{1}+\theta_{2}\right)+2 \vec{l}_{1} \vec{l}_{4} m_{3} \sin \left(\theta_{1}+\theta_{2}\right.$

$\left.+\theta_{3}\right)$

$\frac{\partial E_{K}}{\partial \dot{\theta}_{3}}=\vec{l}_{1} \vec{l}_{2} m_{3} \dot{\theta}_{1} \cos \left(\theta_{1}+\theta_{2}+\theta_{3}\right)+2 \vec{l}_{4} m_{3}\left(\dot{\theta}_{1}+\dot{\theta}_{2}+\dot{\theta}_{3}\right) \sin ^{2}\left(\theta_{1}+\theta_{2}+\right.$

$\left.\theta_{3}\right)+\vec{l}_{1} \vec{l}_{2} m_{3}\left(\dot{\theta}_{1}+\dot{\theta}_{2}+\dot{\theta}_{3}\right) \cos \left(\theta_{1}+\theta_{2}\right)+\vec{l}_{1} \vec{l}_{2} m_{3} \dot{\theta}_{1} \cos \left(\theta_{1}+\theta_{2}\right)+\vec{l}_{2} \vec{l}_{3} m$

${ }_{3}\left(\dot{\theta}_{1}+\dot{\theta}_{2}\right) \cos \theta_{3}+\vec{l}_{1} \vec{l}_{2} m_{3} \dot{\theta}_{1} \cos \left(\theta_{1}+\theta_{2}\right)+\vec{l}_{2} \vec{l}_{3} m_{3}\left(\dot{\theta}_{1}+\dot{\theta}_{3}\right) \cos \theta_{3}+2 \vec{l}_{1} \vec{l}_{4}$

$m_{3} \cos \left(\theta_{1}+\theta_{2}+\theta_{3}\right)$

And

$\frac{d}{d t}\left(\frac{\partial E_{K}}{\partial \dot{\theta}_{1}}\right)=\vec{l}_{2} m_{2}\left(\ddot{\theta}_{1}+\ddot{\theta}_{2}\right)+4\left(\ddot{\theta}_{1}+\ddot{\theta}_{2}\right) \dot{\theta}_{2} \vec{l}_{1} \vec{l}_{2} m_{2} \sin \theta_{2} \cos \theta_{2}+4\left(\ddot{\theta}_{1}\right.$

$\left.+\ddot{\theta}_{2}\right) \vec{l}_{4} m_{3} \sin ^{2}\left(\theta_{1}+\theta_{2}\right)+4\left(\dot{\theta}_{1}+\dot{\theta}_{2}\right)^{3} \vec{l}_{4} m_{3} \cos \left(\theta_{1}+\theta_{2}\right)+2\left(\dot{\theta}_{1}+\dot{\theta}_{2}\right) \vec{l}_{4}$

$m_{3} \sin ^{2}\left(\theta_{1}+\theta_{2}\right)+4\left(\dot{\theta}_{1}+\dot{\theta}_{2}+\dot{\theta}_{3}\right) \vec{l}_{4} m_{3} \sin \left(\theta_{1}+\theta_{2}+\theta_{3}\right)-2 \dot{\theta}^{2} \vec{l}_{1} \vec{l}_{2} m_{2} \sin \theta_{2}-2$

$\left(\dot{\theta}_{1}+\dot{\theta}_{2}\right) \dot{\theta}_{2} \vec{l}_{1} \vec{l}_{2} m_{2} \sin \theta_{1}+2 \ddot{\theta} \vec{l}_{1} \vec{l}_{2} m_{2} \cos \theta_{2}-2 \dot{\theta}^{2} \vec{l}_{1} \vec{l}_{2} m_{2} \sin \theta_{2}+\ddot{\theta}_{\vec{l}} \vec{l}_{2} m_{3}$

$\cos \left(\theta_{1}+\theta_{2}\right)-\dot{\theta}_{1} \vec{l}_{1} \vec{l}_{2} m_{3}\left(\dot{\theta}_{1}+\dot{\theta}_{2}\right) \sin \left(\theta_{1}+\theta_{2}\right)+\vec{l}_{2} m_{3}\left(\ddot{\theta}_{1}+\ddot{\theta}_{2}+\ddot{\theta}_{3}\right) \cos \left(\theta_{1}+\theta_{2}\right)$

$+\vec{l}_{2} m_{3}\left(\dot{\theta}_{1}+\dot{\theta}_{2}+\dot{\theta}_{3}\right)\left(\dot{\theta}_{1}+\dot{\theta}_{2}\right) \cos \left(\theta_{1}+\theta_{2}\right)+\vec{l}_{2} m_{3}\left(\ddot{\theta}_{1}+\ddot{\theta}_{2}\right) \cos \theta_{3}+\vec{l}_{2} m_{3}\left(\dot{\theta}_{1}+\dot{\theta}_{2}\right)$

$\dot{\theta}_{3} \cos \theta_{3}+\vec{l}_{1} \vec{l}_{2} m_{3}\left(\ddot{\theta}_{1}+\ddot{\theta}_{2}+\ddot{\theta}_{3}\right) \cos \theta_{3}+\vec{l}_{2} \vec{l}_{3} m_{3}\left(\ddot{\theta}_{1}+\ddot{\theta}_{2}+\ddot{\theta}_{3}\right) \cos \theta_{3}+\vec{l}_{2} \vec{l}_{3} m_{3}$

$\left(\dot{\theta}_{1}+\dot{\theta}_{2}+\dot{\theta}_{3}\right) \dot{\theta}_{3}+4\left(\ddot{\theta}_{1}+\ddot{\theta}_{2}\right) \vec{l}_{1} \vec{l}_{4} m_{3} \cos \left(\theta_{1}+\theta_{2}\right)+2\left(\dot{\theta}_{1}+\dot{\theta}_{2}\right)^{3} \vec{l}_{1} \vec{l}_{4} m_{3} \cos \left(\theta_{1}+\theta_{2}\right)$

$-2\left(\dot{\theta}_{1}+\dot{\theta}_{2}+\dot{\theta}_{3}\right) \vec{l}_{1} \vec{l}_{4} m_{3} \sin \left(\theta_{1}+\theta_{2}+\theta_{3}\right)$

$\frac{d}{d t}\left(\frac{\partial E_{K}}{\partial \dot{\theta}_{2}}\right)=\vec{l}_{2} m_{2}\left(\ddot{\theta}_{2}+\ddot{\theta}_{1}\right)+2 \vec{l}_{4} m_{3}\left(\dot{\theta}_{1}+\dot{\theta}_{2}\right)\left(\ddot{\theta}_{2}+\ddot{\theta}_{1}\right) \sin ^{2}\left(\theta_{1}+\theta_{2}\right)-4 \vec{l}_{4} m_{3}\left(\dot{\theta}_{1}\right.$

$\left.+\dot{\theta}_{2}\right)^{2}\left(\ddot{\theta}_{2}+\ddot{\theta}_{1}\right) \sin \left(\theta_{1}+\theta_{2}\right) \cos \left(\theta_{1}+\theta_{2}\right)+2 \vec{l}_{4} m_{3}\left(\ddot{\theta}_{1}+\ddot{\theta}_{2}+\ddot{\theta}_{3}\right)\left(\dot{\theta}_{1}+\dot{\theta}_{2}\right) \sin ^{2}\left(\theta_{1}\right.$

$\left.+\theta_{2}\right)+2 \vec{l}_{4} m_{3}\left(\ddot{\theta}_{1}+\ddot{\theta}_{2}\right) \sin ^{2}\left(\theta_{1}+\theta_{2}\right)-4 \vec{l}_{4} m_{3} \dot{\theta}_{1}\left(\dot{\theta}_{1}+\dot{\theta}_{2}\right)^{2} \sin \left(\theta_{1}+\theta_{2}\right) \cos \left(\theta_{1}+\right.$

$\left.\theta_{2}\right)+2 \vec{l}_{1} \vec{l}_{2} m_{3} \ddot{\theta}_{1} \cos \theta_{2}+2 \vec{l}_{1} \vec{l}_{2} m_{2} \dot{\theta}_{1} \dot{\theta}_{2} \cos \theta_{2}+\vec{l}_{1} \vec{l}_{2} m_{3}\left(\ddot{\theta}_{1}+\ddot{\theta}_{2}\right) \cos \left(\theta_{1}+\theta_{2}\right)$

$-\vec{l}_{1} \vec{l}_{2} m_{3}\left(\dot{\theta}_{1}+\dot{\theta}_{2}+\dot{\theta}_{3}\right)\left(\dot{\theta}_{1}+\dot{\theta}_{2}\right) \sin \left(\theta_{1}+\theta_{2}\right)+\vec{l}_{1} \vec{l}_{2} m_{3}\left(\ddot{\theta}_{1}+\ddot{\theta}_{2}+\ddot{\theta}_{3}\right) \cos \theta_{1}-\vec{l}_{1} \vec{l}_{2}$

$m_{3}\left(\dot{\theta}_{1}+\dot{\theta}_{2}+\dot{\theta}_{3}\right) \dot{\theta}_{1} \sin \theta_{1}+\vec{l}_{2} \vec{l}_{3} m_{3}\left(\ddot{\theta}_{1}+\ddot{\theta}_{2}+\ddot{\theta}_{3}\right) \cos \theta_{3}+2 \vec{l}_{1} \vec{l}_{4} m_{3}\left(\dot{\theta}_{1}+\dot{\theta}_{2}\right) \cos \left(\theta_{1}\right.$

$\left.+\theta_{2}\right)+2 \vec{l}_{1} \vec{l}_{4} m_{3}\left(\dot{\theta}_{1}+\dot{\theta}_{2}+\dot{\theta}_{3}\right) \cos \left(\theta_{1}+\theta_{2}\right)+2 \vec{l}_{1} \vec{l}_{4} m_{3}\left(\dot{\theta}_{1}+\dot{\theta}_{2}+\dot{\theta}_{3}\right) \cos \left(\theta_{1}+\theta_{2}+\theta_{3}\right)$ 
$\frac{d}{d t}\left(\frac{\partial E_{K}}{\partial \dot{\theta}_{3}}\right)=\vec{l}_{1} \vec{l}_{2} m_{3} \ddot{\theta}_{1}\left(\dot{\theta}_{1}+\dot{\theta}_{2}+\dot{\theta}_{3}\right)+2 \vec{l}_{4} m_{3}\left(\ddot{\theta}_{1}+\ddot{\theta}_{2}+\ddot{\theta}_{3}\right) \sin ^{2}\left(\theta_{1}+\theta_{2}+\theta_{3}\right)+$

$4 \vec{l}_{4} m_{3}\left(\dot{\theta}_{1}+\dot{\theta}_{2}+\dot{\theta}_{3}\right)^{2} \sin \left(\theta_{1}+\theta_{2}+\theta_{3}\right) \cos \left(\theta_{1}+\theta_{2}+\theta_{3}\right)-\vec{l}_{1} \vec{l}_{2} m_{3}\left(\ddot{\theta}_{1}+\ddot{\theta}_{2}+\ddot{\theta}_{3}\right)($

$\left.\dot{\theta}_{1}+\dot{\theta}_{2}\right) \sin \left(\theta_{1}+\theta_{2}\right)+\vec{l}_{1} \vec{l}_{2} m_{3} \ddot{\theta}_{1} \cos \left(\theta_{1}+\theta_{2}\right)+\vec{l}_{1} \vec{l}_{2} m_{3} \ddot{\theta}_{1} \cos \left(\theta_{1}+\theta_{2}\right)+\vec{l}_{3} \vec{l}_{2} m_{3}$

$\left(\ddot{\theta}_{1}+\ddot{\theta}_{3}\right) \cos \theta_{3}-\vec{l}_{2} \vec{l}_{3} m_{3}\left(\dot{\theta}_{1}+\dot{\theta}_{3}\right) \dot{\theta}_{3} \sin \theta_{3}-\vec{l}_{1} \vec{l}_{4} m_{3} \cos \left(\dot{\theta}_{1}+\dot{\theta}_{2}+\dot{\theta}_{3}\right)$

$\frac{\partial E_{K}}{\partial \theta_{1}}=\vec{l}_{1}\left(\vec{l}_{1}+\vec{l}_{4}+\vec{l}_{5}\right)\left(m_{1}+m_{2}+m_{3}\right) \theta_{1}+\vec{l}_{2}\left(\vec{l}_{3}+\vec{l}_{4}+\vec{l}_{5}\right) m_{2}\left(\theta_{1}+\theta_{2}\right)+\vec{l}_{3}\left(\vec{l}_{3}+\vec{l}_{4}+\vec{l}_{5}\right) m_{3}\left(\theta_{1}+\theta_{2}+\theta_{3}\right)$

$+2 \vec{l}_{4} m_{3} \dot{\theta}_{1}\left(\dot{\theta}_{1}+\dot{\theta}_{2}\right)^{2} \sin \left(\theta_{1}+\theta_{2}\right)-2 \vec{l}_{4} m_{3} \dot{\theta}_{1}\left(\dot{\theta}_{1}+\dot{\theta}_{2}+\dot{\theta}_{3}\right)^{2} \sin \left(\theta_{1}+\theta_{2}+\theta_{3}\right)-\vec{l}_{1} \vec{l}_{2} m_{2} \dot{\theta}_{1}\left(\dot{\theta}_{1}+\dot{\theta}_{2}+\dot{\theta}_{3}\right) \sin$

$\left(\theta_{1}+\theta_{2}\right)+\left(\vec{l}_{4}+\vec{l}_{5}\right)\left(m_{2}+m_{2}+m_{3}\right) \theta_{1}+\left(\vec{l}_{4}+\vec{l}_{5}\right) m_{2}\left(\theta_{1}+\theta_{2}\right)+2 \vec{l}_{1} \vec{l}_{4} m_{3}\left(\dot{\theta}_{1}+\dot{\theta}_{2}\right) \cos \left(\theta_{1}+\theta_{2}\right)+2 \vec{l}_{1} \vec{l}_{4} m_{3}$

$\left(\dot{\theta}_{1}+\dot{\theta}_{2}+\dot{\theta}_{3}\right) \cos \left(\theta_{1}+\theta_{2}\right)$

$\frac{\partial E_{K}}{\partial \theta_{2}}=\vec{l}_{2}\left(\vec{l}_{2}+\vec{l}_{4}+\vec{l}_{5}\right) m_{2}\left(\theta_{1}+\theta_{2}\right)+\vec{l}_{3}\left(\vec{l}_{3}+\vec{l}_{4}+\vec{l}_{5}\right) m_{3}\left(\theta_{1}+\theta_{2}+\theta_{3}\right)+4 \vec{l}_{4} m_{3} \dot{\theta}_{1} \sin \theta_{2}+2 \vec{l}_{4} m_{3} \dot{\theta}_{1}\left(\dot{\theta}_{1}+\dot{\theta}_{2}\right)^{2} \sin$

$\left(\theta_{1}+\theta_{2}\right)+2 \vec{l}_{4} m_{3} \dot{\theta}_{1}\left(\dot{\theta}_{1}+\dot{\theta}_{2}+\dot{\theta}_{3}\right)^{2} \sin \left(\theta_{1}+\theta_{2}+\theta_{3}\right)-2 \vec{l}_{1} \vec{l}_{2} m_{3} \dot{\theta}_{1}\left(\dot{\theta}_{1}+\dot{\theta}_{2}\right) \sin \theta_{2}-2 \vec{l}_{1} \vec{l}_{2} m_{3} \dot{\theta}_{1}\left(\dot{\theta}_{1}+\dot{\theta}_{2}\right) \sin \theta_{2}-\left(\vec{l}_{4}\right.$

$\left.+\vec{l}_{5}\right) m_{3} \dot{\theta}_{1}\left(\theta_{1}+\theta_{2}\right)+2 \vec{l}_{1} \vec{l}_{4} m_{3} \dot{\theta}_{1}\left(\dot{\theta}_{1}+\dot{\theta}_{2}+\dot{\theta}_{3}\right) \cos \left(\theta_{1}+\theta_{2}\right)-\left(\vec{l}_{4}+\vec{l}_{5}\right) m_{3} \dot{\theta}_{1}\left(\theta_{1}+\theta_{2}\right)+2 \vec{l}_{1} \vec{l}_{4} m_{3} \dot{\theta}_{1} \cos \left(\theta_{1}+\theta_{2}\right)+$

$2 \vec{l}_{3} \vec{l}_{4} m_{3}\left(\dot{\theta}_{1}+\dot{\theta}_{1}+\dot{\theta}_{2}\right) \cos \left(\theta_{1}+\theta_{2}+\theta_{3}\right)$

(16)

$\frac{\partial E_{K}}{\partial \theta_{3}}=\vec{l}_{3}\left(\vec{l}_{3}+\vec{l}_{4}+\vec{l}_{5}\right)\left(\theta_{1}+\theta_{2}+\theta_{3}\right)-2 \vec{l}_{4} m_{3}\left(\dot{\theta}_{1}+\dot{\theta}_{2}+\dot{\theta}_{3}\right)^{2} \sin \left(\theta_{1}+\theta_{2}+\theta_{3}\right)-\vec{l}_{2} \vec{l}_{3} m_{3}\left(\dot{\theta}_{1}+\dot{\theta}_{2}\right)$

$\left(\dot{\theta}_{1}+\dot{\theta}_{2}+\dot{\theta}_{3}\right) \sin \theta_{3}-2 \vec{l}_{3} \vec{l}_{4} m_{3}\left(\dot{\theta}_{1}+\dot{\theta}_{2}+\dot{\theta}_{3}\right) \cos \left(\theta_{1}+\theta_{2}+\theta_{3}\right)$

potential energy of System

$E_{p}=\left(\vec{l}_{1}+\vec{l}_{4}\right) m_{1} g \cos \theta_{1}+\left(\vec{l}_{2}+\vec{l}_{4}\right) m_{2} g \cos \left(\theta_{1}+\theta_{2}\right)+\left(\vec{l}_{3}+\vec{l}_{4}\right) m_{3} g \cos \left(\theta_{1}+\theta_{2}+\theta_{3}\right)$

$\frac{\partial E_{P}}{\partial \theta_{1}}=\vec{l}_{1} \vec{l}_{4} m_{1} g \dot{\theta}_{1} \sin \theta_{1}$

$\frac{\partial E_{P}}{\partial \theta_{2}}=\vec{l}_{2} \vec{l}_{4} m_{2} g \dot{\theta}_{2} \sin \left(\theta_{1}+\theta_{2}\right)$

$\frac{\partial E_{P}}{\partial \theta_{3}}=\vec{l}_{3} \vec{l}_{4} m_{3} g \dot{\theta}_{3} \sin \left(\theta_{1}+\theta_{2}+\theta_{3}\right)$

Substituting Lagrange equation below (10) for above equations

Lagrange equation is

$\frac{d}{d t}\left(\frac{\partial E_{K}}{\partial \dot{q}_{i}}\right)-\frac{\partial E_{K}}{\partial q_{i}}+\frac{\partial E_{P}}{\partial q_{i}}=F_{i},(\mathrm{i}=1,2, \ldots, \mathrm{n})$

Here $E_{K}$ is kinetic of system;

$E_{P}$ is potential energy of system;

$q_{i}$ is generalized coordinate, it is a group of independent parameters that can define mechanical system movement;

$F_{i}$ is generalized force, when $q_{i}$ is a angular displacement it a torque, when $q_{i}$ is linear displacement it a force; $\mathrm{n}$ is system generalized coordinate. 
System generalized force

Supposed that $\mathrm{Fk}(\mathrm{k}=1,2, . ., \mathrm{m})$ and $\mathrm{Mj}(\mathrm{j}=1,2, . ., \mathrm{n})$ is force and torque acting on system. Its power is

$P=\sum_{k=1}^{m}\left(F_{k} v_{k} \cos \alpha_{k}\right)+\sum_{j=1}^{n}\left( \pm M_{j} \omega_{j}\right)$

Here $\omega \mathrm{j}$ : angular velocity acting on component with $\mathrm{Mj}$;

vk: the velocity in force Fk point of action; (the syntropy + , reverse direction -)

ak: angle between $\mathrm{Fk}$ and vk

When generalized coordinates is $\varphi$ angular displacement generalized force=equivalent torque Me.

$\delta W_{2}=\sum_{k=1}^{m}\left(F_{k} \delta v_{k} \cos \alpha_{k}\right)+\sum_{j=1}^{n}\left( \pm M_{j} \delta \omega_{j}\right)$

Here $a_{K}$ is zero; $F_{K}=200 \mathrm{~N} ; v_{K}=0.2 \sim 0.3 \mathrm{~m} / \mathrm{s} ; \omega_{j}=; 20 \sim 30 \% M_{j}=20 \sim 30 \mathrm{Nm} . \delta \varphi_{j}$ is virtual angular displacement; $\delta s_{k}$ is virtual displacement.

Supposing that

$$
\begin{aligned}
& \delta s_{K}=\frac{\partial s_{K}}{\partial q_{1}} \delta q_{1}+\frac{\partial s_{K}}{\partial q_{2}} \delta q_{2} \\
& \delta \varphi_{K}=\frac{\partial \varphi_{j}}{\partial q_{1}} \delta q_{1}+\frac{\partial \varphi_{j}}{\partial q_{2}} \delta q_{2}
\end{aligned}
$$

Replace equation below with above two equations

$$
\left\{\begin{array}{l}
F_{1}=\sum_{k=1}^{m}\left[F_{k} \frac{\partial s_{K}}{\partial q_{1}} \cos \alpha_{k}\right]+\sum_{j=1}^{n}\left[M_{J} \frac{\partial \varphi_{J}}{\partial q_{1}}\right] \\
F_{2}=\sum_{k=1}^{m}\left[F_{k} \frac{\partial s_{K}}{\partial q_{2}} \cos \alpha_{k}\right]+\sum_{j=1}^{n}\left[M_{J} \frac{\partial \varphi_{J}}{\partial q_{2}}\right]
\end{array}\right.
$$

This is generalized force equation.

\section{DISCUSSIONS}

As seen in Table 1 the parameter in robot arm is listed [6, 7]. Here $\theta_{1}, \theta_{2}, \theta 3$ is the $\operatorname{arm}_{1}, \operatorname{arm}_{2}, \operatorname{arm}_{3}$ angle respectively. $\mathrm{l}_{1}, \mathrm{l}_{2}, \mathrm{l}_{3}$ is arm length. $\mathrm{m}_{1}, \mathrm{~m}_{2}, \mathrm{~m}_{3}$ is arm mass. Number is arm label. According to these parameters the below curves are gained as below in Figure $3 \& 4$. As seen in Figure $3(a \sim c)$ the force of arm 1 will increase with the angular speed and acceleration increasing that expresses the proportional relation between them fitting to Newton theory well. That says that angular speed raises the acceleration meantime the later raise the force too. They all distributes into sinusoidal continuous wave that forms semiwave with $90^{\circ}$. The force may increase from $15 \mathrm{~N}$ to $15 \mathrm{KN}$ and $18 \mathrm{KN}$ with $F_{3}, F_{1}$ and $F_{2}$ as seen in Figure 4. Among them $F_{3}$ is the least one and $F_{1}$ is the biggest one. The effect factor turn is $F_{1}>F_{2}$ $>F_{3}$ at the angular acceleration of $20 \% \mathrm{~s}^{2}$. So the $F_{1}$ is important one attained 1.8Tons and $F_{2}$ is second attains 1.5 tons while $F_{3}$ is neglected. From these value it is observed that $F_{1}$ is prior one to ensure the strength and fatigue life then $F_{2}$ is second one to estimate its strength whilst $\mathrm{F}_{3}$ may be neglected in five freedoms. In contrast to above in Figure 3 in three freedoms the force $\mathrm{F}_{1}$ is about $18 \mathrm{KN}$ ie. 1.8 tons which is the biggest need to be checked the strength estimation and then $F_{3}$ is $75 \mathrm{~N}$ and at last $F_{2}$ is $16 \mathrm{~N}$ which are neglected here. The effect turn is $F_{1}>F_{3}>F_{2}$.

Table-1: Parameters of robot arms

\begin{tabular}{|l|l|l|l|}
\hline Items & Value & Item & Value \\
\hline $11 / \mathrm{m}$ & 0.55 & $\dot{\theta}_{1} / \%$ & $30 \sim 60$ \\
\hline $12 / \mathrm{m}$ & 0.5 & $\dot{\theta}_{2} / \%$ & $30 \sim 60$ \\
\hline
\end{tabular}




\begin{tabular}{|l|l|l|l|}
\hline $13 / \mathrm{m}$ & 0.3 & $\dot{\theta}_{3} / \%$ & $30 \sim 60$ \\
\hline $\mathrm{m} 1 / \mathrm{N}$ & 7.7 & $\ddot{\theta}_{1} / \% \mathrm{~s}^{2}$ & 20 \\
\hline $\mathrm{m} 2 / \mathrm{N}$ & 6.6 & $\ddot{\theta}_{2} / \% \mathrm{~s}^{2}$ & 20 \\
\hline $\mathrm{m} 3 / \mathrm{N}$ & 4.0 & $\ddot{\theta}_{3} / \% \mathrm{~s}^{2}$ & 20 \\
\hline
\end{tabular}

As seen in Figure 3 the force may increase as arm 1 angle increases whilst it may increase if angular speed increases in three freedoms. Meantime it will decrease if angular acceleration increases in Figure 3(d). The maximum is $18 \mathrm{KN}$ in Figure 3(a) if angular speed is $20 \% \mathrm{~s}$ and acceleration is $20 \% \mathrm{~s}^{2}$ so this point will be checked to ensure the robotic arm strength. There is big distance to attain $3 \sim 5 \mathrm{KN}$ between the conditions. The effective factor turn to the force is $\mathrm{F}_{1}>\mathrm{F}_{3}>\mathrm{F}_{2}$ in three freedoms.
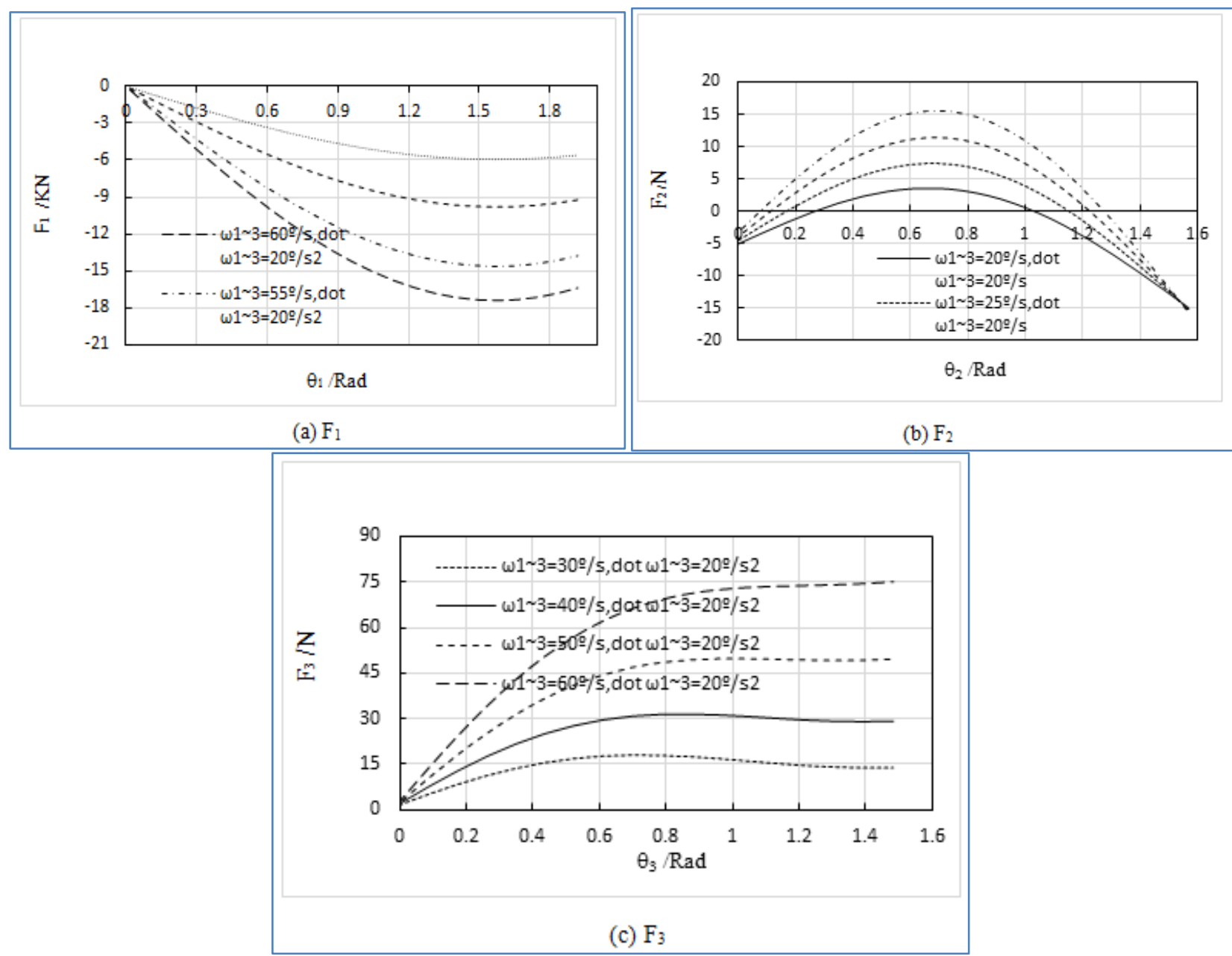

Fig-3: The curve of force and angle with various angular speed and acceleration at angular acceleration of $20^{\circ} / \mathrm{s} 2$ in three freedoms of robot arm.

In the modeling of five freedoms in movement of robotic arm the kinetic equation is established according to Lagrange formula based on three freedoms robotic arm. It compensates the blank in four freedoms and one impulsion on robot. It is found that the first and second solution is complicated and long the whole equations is concise than the traditional equation. This is a blank in five freedoms which can shorten the whole numerical computation a lot. Referring to the important occasion the kinetic equation will only be computed on three freedoms according to this study. 


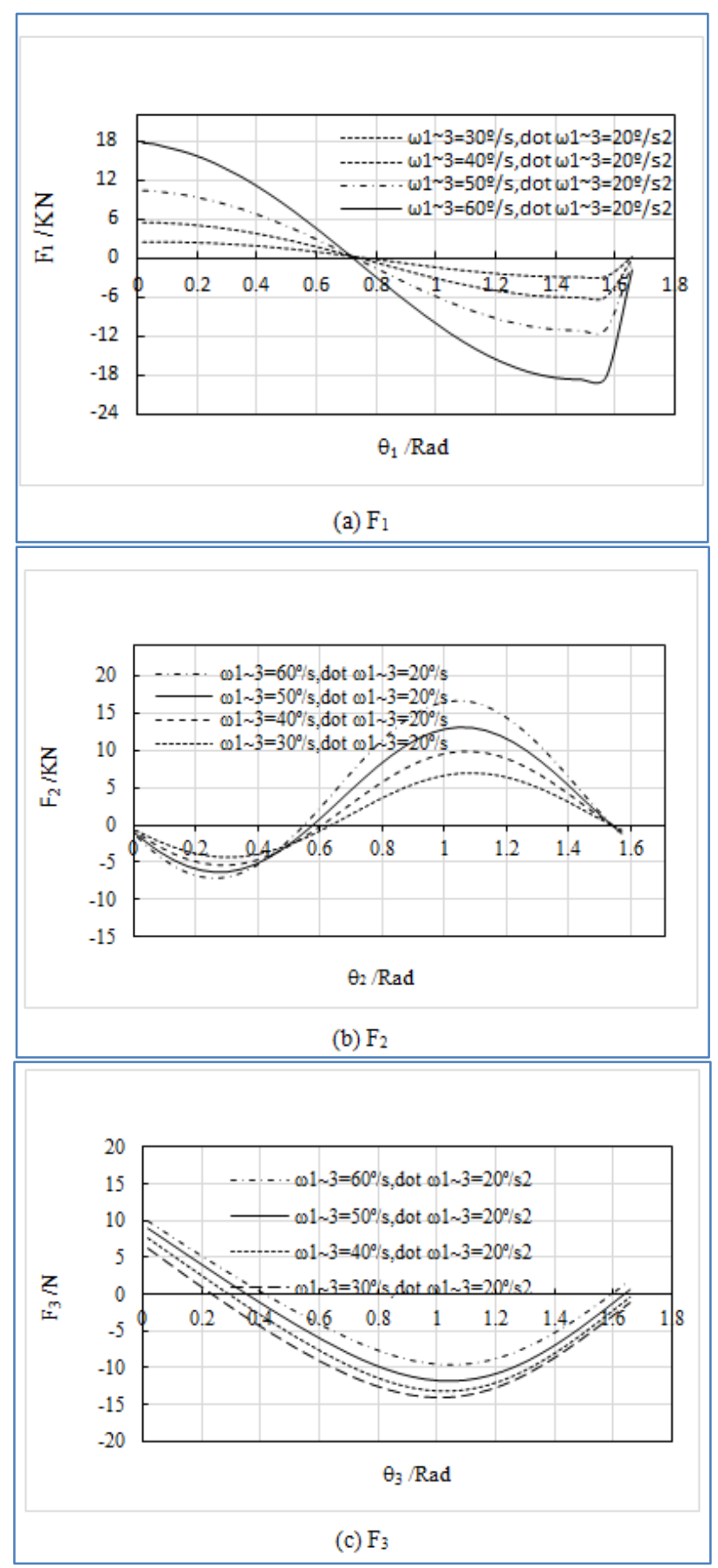

Fig-4: The curve of force and angle with various angular speed and acceleration at angular acceleration of $20 \%$ in five freedoms of robot arm.

It is suggested that the big arm happens when angular speed and acceleration is big. So that the reasonable parameters are chosen to design and estimate their properties is important. Not to choose big angular speed and acceleration is key in order to increase the capability and property that may increase the whole cost as well.

Overview the computation is shorter than the five freedoms traditional one. The solution is easy to use in software like Excel and Origin. The result is satisfactory and precise to be adopted to numerical simulation so the five freedoms method based on three freedoms is feasible.

\section{CONCLUSIONS}

1. There is big distance to attain $5 \mathrm{KN}$ between the conditions. The effective factor turn to the force is $\mathrm{F}_{1}>\mathrm{F}_{3}>\mathrm{F}_{2}$ in three freedoms. 
2. The force may increase from $15 \mathrm{~N}$ to $15 \mathrm{KN}$ and $18 \mathrm{KN}$ with $\mathrm{F}_{3}, \mathrm{~F}_{2}$ and $\mathrm{F}_{1}$ in five freedoms. Among them $\mathrm{F}_{3}$ is the least one and $F_{1}$ is the biggest one. The effect factor turn is $F_{1}>F_{2}>F_{3}$. so the $F_{1}$ and $F_{2}$ is important one while $F_{3}$ is neglected.

\section{REFERENCES}

1. Zhang, C.E. (2008). Machinery dynamics. Higher Educational Press, 96

2. Xiong, Y., Tang, L. (1999). The base of Robotic Technology. Huazhong University of Technology press, 89-90

3. Run, X. (2020). The Dynamic Equation on Hammer with Lagrange in Robotic Arm, Social Science learning Education Journal, August, 5(8), 297-300,https://doi. org/10. 15520/sslej.v5i08.2703

4. Li, Doyi. Chen, L., Shang, X., Wang, Z., Wang, W., Yang, X.(2019). Structrue design of pineapple picking manipulator[J], Agricultural Engineering, 9(2); 1-2

5. Run, X., Boyong, H. (2021). A Simulation between Torque and Angle with Speed on Five Freedoms of Robot Mechanical Arm in Multibody Systems, Saudi Journal of Civil Engineering, 5(5); 91-93.DOI: 10.36348/sjce.2021.v05i05.003

6. Pengju, Y. (2012). Base on Dynamics Analysis \& Precise Design with Mechanical Arm of Arthritis Rub [D], Master Degree Thesis, Jiangsu University of Technology, 3; 18

7. Zhihua, L.I., Chenjia, W.U. (2020). The Dynamics Solving Method of Flexible Joint Robot Based on Quantized State System[J], Journal of Mechanical Engineering, 56(3); 122 